\title{
Acute new-onset symptomatic seizures in the context of mild COVID-19 infection
}

\author{
Maria Gaughan $^{1}\left[\right.$ D Sean Connolly ${ }^{2} \cdot$ Shamindra Direkze $^{3} \cdot$ Justin A. Kinsella ${ }^{1}$
}

Received: 28 July 2020 / Revised: 1 September 2020 / Accepted: 2 September 2020 / Published online: 10 September 2020

(c) Springer-Verlag GmbH Germany, part of Springer Nature 2020

\section{Dear Sirs,}

In the midst of the COVID-19 global pandemic, two patients with no previous history of seizures presented to our institution with acute symptomatic seizures in the context of mild respiratory SARS-CoV-2 infection.

\section{Case 1}

An 87-year-old male presented to the Emergency Department (ED) at our hospital following a generalised tonic-clonic seizure, with another 30-s convulsion witnessed in ED.

During a recent admission at another hospital for treatment of cellulitis, hoarseness prompted COVID-19 testing. RT-PCR amplification of SARS-Cov-2 nucleic acid from a nasopharyngeal swab was positive. He had remained otherwise asymptomatic and tested negative for SARS-CoV-2 prior to discharge. He had a history of traumatic brain injury, 32 years previously, from which he made a full recovery.

Examination in ED revealed drowsiness and disorientation with no lateralising clinical signs.

Urgent brain CT revealed encephalomalacia involving the inferior frontal and bilateral anterior temporal lobes consistent with previous trauma. CXR was normal. Laboratory investigations are outlined in Table 1. RT-PCR amplification of SARS-Cov-2 nucleic acid from a nasopharyngeal swab

Maria Gaughan

Maria.gaughan@svhg.ie

1 Department of Neurology, University College Dublin, St. Vincent's University Hospital, Elm Park, Dublin 4, Ireland

2 Department of Clinical Neurophysiology, St. Vincent's University Hospital and University College Dublin, Dublin, Ireland

3 Department of Gastroenterology, St. Vincent's University Hospital, Elm Park, Dublin 4, Ireland was positive. CSF was not performed due to rapid clinical recovery. The patient remained clinically well with no respiratory symptoms throughout admission.

EEG was performed following discharge. This demonstrated intermittent fronto-temporal dysfunction maximal on the right side, compatible with the known imaging abnormalities.

\section{Case 2}

A 77-year-old female presented to ED following a prolonged first generalized tonic-clonic seizure. A further generalised convulsion in the ambulance was terminated with $4 \mathrm{mg}$ intravenous (IV) Lorazepam after 3 min.

Collateral history revealed malaise for two days prior to presentation, but no respiratory symptoms. She had no relevant past medical history. She was high functioning at baseline, working in higher education as a course director.

Urgent noncontrast $\mathrm{CT}$ brain and $\mathrm{CT}$ angiography was normal. CXR demonstrated bibasal infiltrates. RT-PCR amplification of SARS-Cov-2 nucleic acid from a nasopharyngeal swab was positive. CSF constituents were normal (Table 1) and SARS-CoV-2 in CSF was not detected. MRI Brain was normal.

The patient had a prolonged encephalopathy with disorientation and bradyphrenia resulting in a protracted admission. EEG was performed three weeks following initial presentation and demonstrated significant bilateral cerebral dysfunction without any epileptiform abnormalities (Fig. 1). Montreal Cognitive Assessment (MOCA) performed four months post hospitalization was $24 / 30$, suggesting persistent cognitive deficit. 
Table 1 Initial laboratory investigations for each patient following presentation with seizures

\begin{tabular}{lllll}
\hline Variable & Units & References & Case 1 & Case 2 \\
\hline White Cell Count & $10^{9} / \mathrm{L}$ & $3.5-11$ & 11.6 & 11.9 \\
Lymphocytes & $10^{9} / \mathrm{L}$ & $1-4$ & 0.6 & 0.9 \\
Neutrophils & $10^{9} / \mathrm{L}$ & $2-8$ & 9.6 & 8.9 \\
SARS-CoV2 RNA & & & Detected & Detected \\
Ferritin & ug/L & $13-150$ & 221 & 657 \\
LDH & $\mathrm{ng} / \mathrm{L}$ & $240-480$ & 450 & 469 \\
Troponin & $\mathrm{ng} / \mathrm{L}$ & $3-14$ & 36 & 37 \\
CSF WCC & Per cmm & $0-5$ & N/A & 3 \\
CSF Protein & $\mathrm{g} / \mathrm{L}$ & $0.15-0.45$ & N/A & 0.3 \\
CSF Glucose & $\mathrm{mmol} / \mathrm{L}$ & & N/A & 3.7 \\
CSF Gram Stain & & & N/A & Negative \\
CSF Viral PCR & & & N/A & Not detected \\
$\quad$ HSV/VZV/Enter- & & & & \\
$\quad$ & & & N/A & Not detected \\
SARS CoV2 (CSF) & & & & \\
\hline
\end{tabular}

\section{Discussion}

We describe two patients presenting acutely with newonset seizures in the context of COVID-19 infection. While one patient had a history of traumatic brain injury 32 years previously, this was not accompanied by or followed by seizures and the second patient had no known risk factors for seizures. In this case, severity of COVID 19 infection-in terms of hypoxia, multi-organ failure or marked metabolic derangement, suggested as potential confounders [1], did not appear to be a causative factor. The severe and prolonged cognitive deficit in the second patient was notable given the relatively mild clinical course of her COVID-19 infection. We presume she developed a form of COVID-19-related encephalopathy with persistent EEG abnormalities noted. We note that neither patient had radiological or biochemical evidence of direct CNS COVID-19 involvement.

While initial reporting suggested that neurological complications were uncommon in COVID-19 infection, seizures as the presenting complaint are increasingly reported $[2,3]$. Rarely, severe neurological presentations manifest in those with mild infection $[4,5]$.

The pathological mechanism underlying multisystem involvement in SARS-Co-V2 is unclear. The possibility of direct invasion of the neural parenchyma via the olfactory bulb, resulting in neuronal death without widespread inflammation has been postulated [6]. The second pathogenic theory of a proinflammatory cascade in the context of a cytokine storm seems less likely here in the context of mild COVID-19 infection. The pathophysiological mechanisms involved remain elusive, particularly given the rarity of SARS-CoV-2 detection in the CSF [7]. Lack of access to detailed investigations due to resource restrictions will limit data available in retrospective reporting.
Fig. 1 Electrocephalogram (EEG) in a 77-year-old female, awake and confused (Case 2); 10-20 International system of scalp electrode placement; bipolar montage; $C G$ electrocardiogram, $E O G$ electrooculogram, $\mu V$ microVolts, $H z$ Hertz, the tracing depicts recurrent excess of widespread bilateral theta and delta activity

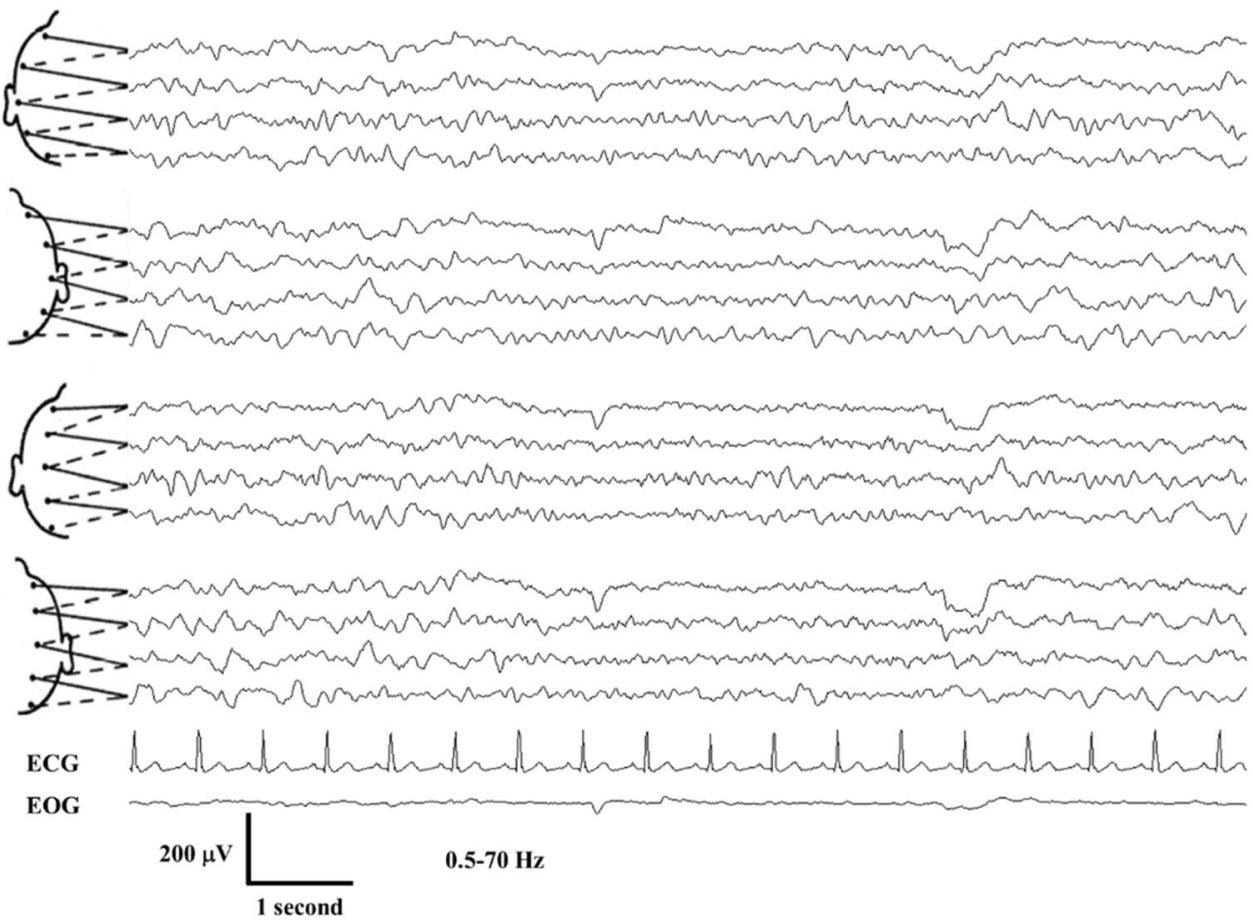


Author contributions MG: St. Vincent's University Hospital and University College Dublin, Design and conceptualized study; analyzed the data; drafted the manuscript for intellectual content; SC, SD: St. Vincent's University Hospital and University College Dublin, Data collection and analysis, drafting and revision of manuscript; JK: St. Vincent's University Hospital and University College Dublin, Study design, drafting and revision of manuscript.

Funding Not applicable.

Data availability Clinical data is available for review.

\section{Compliance with ethical standards}

Conflicts of interests Not applicable.

Ethical approval Not applicable.

Consent to participate The patients involved have voluntarily signed consent to allow for reporting of their data.

Consent for publication The patients involved have given informed consent for publication of their data.

\section{References}

1. Asadi-Pooya AA (2020) Seizures associated with coronavirus infections. Seizure 79:49-52. https://doi.org/10.1016/j.seizu re.2020.05.005
2. Anand P, Al-Faraj A, Sader E, Dashkoff J, Abdennadher M, Murugesan R, Cervantes-Arslanian AM, Daneshmand A (2020) Seizure as the presenting symptom of COVID-19: a retrospective case series. Epilepsy Behav 112:107335. https://doi.org/10.1016/j. yebeh.2020.107335

3. Lyons S, O'Kelly B, Woods S, Rowan C, Brady D, Sheehan G, Smyth S (2020) Seizure with CSF lymphocytosis as a presenting feature of COVID-19 in an otherwise healthy young man. Seizure 80:113-114. https://doi.org/10.1016/j.seizure.2020.06.010

4. Munz M, Wessendorf S, Koretsis G, Tewald F, Baegi R, Krämer S, Geissler M, Reinhard M (2020) Acute transverse myelitis after COVID-19 pneumonia. J Neurol 267(8):2196-2197. https://doi. org/10.1007/s00415-020-09934-w

5. Swarz JA, Daily S, Niemi E, Hilbert SG, Ibrahim HA, Gaitanis JN (2020) COVID-19 infection presenting as acute-onset focal status epilepticus. Pediatr Neurol 112:7. https://doi.org/10.1016/j.pedia trneurol.2020.07.012

6. Netland J, Meyerholz DK, Moore S, Cassell M, Perlman S (2008) Severe acute respiratory syndrome coronavirus infection causes neuronal death in the absence of encephalitis in mice transgenic for human ACE2. J Virol 82(15):7264-7275. https://doi. org/10.1128/jvi.00737-08

7. Espíndola OdM, Siqueira M, Soares CN, Lima MASDd, Leite ACCB, Araujo AQC, Brandão CO, Silva MTT (2020) Patients with COVID-19 and neurological manifestations show undetectable SARS-CoV-2 RNA levels in the cerebrospinal fluid. Int J Infectious Diseases 96:567-569. https://doi.org/10.1016/j. ijid.2020.05.123 